\title{
A Statistical Analysis of Superior Orbital Fissure Width in Korean Adults using Computed Tomography Scans
}

Yongsik Park, Youngjoon Kim

Department of Plastic and Reconstructive Surgery, Sanggye Paik Hospital, Inje University College of Medicine, Seoul, Korea

No potential conflict of interest relevant to this article was reported.

\begin{abstract}
Background: The superior orbital fissure is a small area that connects the middle cranial fossa and the orbit. Many studies have measured the size of the superior orbital fissure. However, there is no standard value for the size of the superior orbital fissure. Therefore, we conducted this study to provide the average size of the superior orbital fissure in Korean adults. Methods: We measured the widths of the superior orbital fissures of 142 patients using computed tomography scans. Because the width of the superior orbital fissure varies at different locations, we measured the superior orbital fissure width at the level of the optic canal.

Results: In the males, the width of the superior orbital fissure on both sides was $3.79 \pm 0.93$ $\mathrm{mm}$, and these values were $3.79 \pm 0.96 \mathrm{~mm}$ for the left side and $3.783 \pm 0.92 \mathrm{~mm}$ for the right side. In the females, the widths of the superior orbital fissures were $3.62 \pm 1.35 \mathrm{~mm}$ on the left side, $3.69 \pm 1.18 \mathrm{~mm}$ on the right side, and $3.65 \pm 1.26 \mathrm{~mm}$ across both sides.

Conclusion: There were no significant differences between the males and females or between the left and right sides. The present study suggests that we may accept the hypothesis that a congenitally narrow superior orbital fissure may be a risk factor for the superior orbital fissure syndrome. Surgeons should take precaution with patients who have narrow superior orbital fissures during the perioperative period.
\end{abstract}

Keywords: Cranial nerves / Orbit / Tomography, X-ray computed

\section{INTRODUCTION}

The superior orbital fissure is a small space that connects the middle cranial fossa and the orbit [1-3]. Many nerves including cranial nerves (III, IV, V, and VI), and vessels pass through this fissure $[1,2]$. The superior orbital fissure syndrome (SOFS) is a complex of impaired functions of the cranial nerves due to compression or disruption of the superior orbital fissure. The SOFS may result from various causes that include inflammation, infection, neoplasm, iatrogenic/traumatic factors and vascular problems $[1,3,4]$. The SOFS has many clinical features including the loss of sensa-

\section{Correspondence: Youngjoon Kim}

Department of Plastic and Reconstructive Surgery, Sanggye Paik Hospital, Inje University College of Medicine, 1342 Dongil-ro, Nowon-gu, Seoul 01757, Korea

E-mail: S2639@paik.ac.kr

Received February 20, 2017 / Revised April 28, 2017 / Accepted April 30, 2017 tion over the forehead, edema in the periorbital region, proptosis, dilation of the pupil, ptosis, ophthalmoplegia, loss of the corneal reflex, loss of the direct light reflex and lacrimal hypo-secretion [1-4]. Many previous studies have investigated the size of the superior orbital fissure. One previous study suggested that a congenitally narrow superior orbital fissure is a risk factor for the SOFS [4]. To use this risk factor in the clinic as a diagnostic tool, the average size of the superior orbital fissure needs to be defined which we did the present study.

\section{METHODS}

This was a retrospective cohort study. Between November 2011 and May 2014, the computed tomography (CT) scans of patients over the age of 20 years who visited our department were exam- 
ined. These patients underwent CT scans to evaluate facial trauma. Of 211 patients, 63 patients who diagnosed with craniofacial fractures were excluded from this study. 6 patients with prior history of facial fractures, as well as congenital cranial or facial abnormalities were also excluded, leaving 142 patients for this study. Because the width of the superior orbital fissure varies across locations, we measured the superior orbital fissure widths on axial view CT scans at the level of the optic canal level using m-view 5, 4 (Marosis, Marotech, Seoul, Korea) according to a previous study by Fujiwara et al. [4] (Fig. 1). We measured the superior orbital fissures on both sides at different levels using CT scans when the CT scans were distorted such that the optic canal levels varied between the side. From the obtained data, the mean, standard deviation and 95\% coefficient intervals of the widths of the superior orbital fissures were calculated. Using the independent two-sample t-test, we examined the differences between the left- and right-side superior orbital fissure widths. We also analyzed the difference between men and women. Additionally, we compared the man superior orbital fissure width with those that have been reported in studies conducted in other countries. The criterion for significance for all tests was set 0.05 . All statistical tests were performed with PASW statistics ver. 18.0 (SPSS Inc., Chicago, IL, USA), statistical program.

\section{RESULTS}

The patients included 69 males and 73 females. Their mean age was

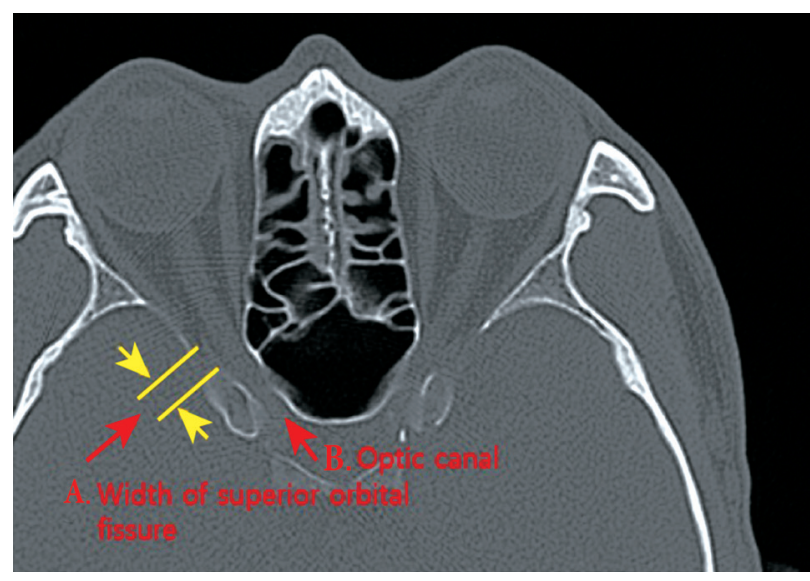

Fig. 1. Measurement of the superior orbital fissure width using computed tomography scans.
$35.8 \pm 13.98$ years (range, $20-68$ years). The widths of the superior orbital fissures in these Korean adults were $3.73 \pm 1.06 \mathrm{~mm}$ on right (maximum, 6.67; minimum, 1.95), 3.70 $\pm 1.17 \mathrm{~mm}$ on the left (maximum, 7.47; minimum, 1.35 ) and $3.72 \pm 1.11 \mathrm{~mm}$ across both sides.

In the males, the widths of the superior orbital fissures were $3.78 \pm 0.92 \mathrm{~mm}$ on the right (maximum, 6.53; minimum, 2.04) $3.79 \pm 0.96 \mathrm{~mm}$ on the left side (maximum, 6.98; minimum, 2.04; $p=0.964$ ) and $3.79 \pm 0.93$ across the both sides.

The widths of the superior orbital fissures of the female patients were $3.69 \pm 1.18 \mathrm{~mm}$ on the right (maximum, 6.67; minimum, 1.95). $3.62 \pm 1.34 \mathrm{~mm}$ on the left (maximum, 7.47; minimum, 1.35; $p=0.740$ ) and $3.65 \pm 1.26 \mathrm{~mm}$ across both sides (Table 1 ).

There was no significant difference between the left and right sides in either the males $(p=0.964)$ or the females $(p=0.740)$ There was also no significant difference between male and female $(p=0.297)$.

\section{DISCUSSION}

The Superior orbital fissure syndrome is a rare complication that occurs in association with craniofacial trauma [3-5], The complication is characterized by ophthalmoplegia, ptosis, proptosis and hypoesthesia in the forehead and upper eyelid regions [5]. Direct bony compression of the cranial nerves that pass the superior orbital fissure due to facial trauma is known to occur, immediately $[2,3,5]$. However, in many cases, the compression effect of a hematoma or edema of the orbital soft tissue can occur following facial trauma surgery $[4,6]$. In the latter case, the patients are usually normal and do not exhibit symptoms or signs of the superior orbital fissure syndrome prior to surgery. Therefore, it is important to predict whether this syndrome will occur in patients who un-

Table 1. The mean widths of the superior orbital fissures according to gender and side

\begin{tabular}{lccc} 
Variable & Total $(n=142)$ & Male $(n=69)$ & Female $(n=73)$ \\
Both & $3.72 \pm 1.11$ & $3.79 \pm 0.93$ & $3.65 \pm 1.26$ \\
Left & $3.70 \pm 1.17$ & $3.79 \pm 0.96$ & $3.62 \pm 1.34$ \\
Right & $3.73 \pm 1.06$ & $3.78 \pm 0.92$ & $3.69 \pm 1.18$ \\
$p$-value & $0.807^{\text {a) }}$ & $0.964^{\text {a) }}$ & $0.740^{\text {a) }}$ \\
\hline
\end{tabular}

Values are presented as mean \pm standard deviation $(\mathrm{mm})$.

a) No significant differences $(p>0.05)$. 
dergo trauma surgery [7]. In our experience, delayed superior orbital fissure syndrome occurred in patients with congenitally small superior orbital fissure width those who underwent surgery for blow-out fracture, this supports the argument of this study [7].

There are many factors that are predictive of the risk of the superior orbital fissure syndrome, and the size of the superior orbital fissure is one of these risk factors [4]. Therefore, numerous studies have evaluated the size of the superior orbital fissure. Reymond et al. [2] directly measured the maximum lengths and widths of the superior orbital fissures of the skulls of 100 adult cadavers. Burdan et al. [8] estimated the areas of the superior orbital fissures of Caucasian patients using CT scans with the automatic field setting. These authors estimated the areas of both superior orbital fissures using 3D scans. Fujiwara et al. [4] investigated the width of the superior orbital fissure using cadavers and computed tomography with patients with intact superior orbital fissures. In this study, no significant differences between the widths of the superior orbital fissure as assessed using CT scans and in the cadavers were found.

Therefore, the width of the superior orbital fissure as measured using CT scans reflects the actual width of the superior orbital fissure [4]. These authors suggested that a congenitally narrow superior orbital fissure (less than $1.60 \mathrm{~mm}$ ) is a risk factor for the superior orbital fissure syndrome [4]. Previously, there were no data regarding superior orbital fissure size in the Korean population. We opted to utilize Fujiwara's method due to its simplicity and convenience.

The present study suggests that we should accept the hypothesis that a narrow superior orbital fissure (less than $1.60 \mathrm{~mm}$ ) may be a risk factor for the superior orbital fissure syndrome. Edema of the soft tissue in the orbit may be more compressed in people with narrow superior orbital fissures. Therefore, surgeons should be careful with patients who have congenitally narrow superior orbital fissures.

The significance of the study is the finding that the average superior orbital fissure width in Korean adults is similar to the values reported in previous studies. We may collect additional data to further define the risk factor of the superior orbital fissure width in terms of the superior orbital fissure syndrome. There are limitations to this study. We did not statistically compare our data with the Japanese data because the raw Japanese data were not available. Additionally, the superior orbital fissure displays various shapes. The width of the superior orbital fissure does not completely reflect the size of the superior orbital fissure, and the capacity of the orbit varies slightly across patients. Thus, we need to examine the types of superior orbital fissure using $3 \mathrm{D} \mathrm{CT}$ scans $[4,9]$.

In conclusion, we have provided a mean width of the superior orbital fissures in Korean adults. We will use these data for the facial bone fracture surgery. There were no differences between the superior orbital fissure widths of males and females. Additionally, there was no difference between the widths of the right and the left superior orbital fissure.

\section{REFERENCES}

1. Taylor KH, Mizen KD, Spencer N. Isolated fracture of the superior orbital fissure. Br J Oral Maxillofac Surg 2010;48:178-9.

2. Reymond J, Kwiatkowski J, Wysocki J. Clinical anatomy of the superior orbital fissure and the orbital apex. J Craniomaxillofac Surg 2008;36:346-53.

3. Rai S, Rattan V. Traumatic superior orbital fissure syndrome: review of literature and report of three cases. Natl J Maxillofac Surg 2012;3: 222-5.

4. Fujiwara T, Matsuda K, Kubo T, Tomita K, Yano K, Hosokawa K. Superior orbital fissure syndrome after repair of maxillary and naso-orbito-ethmoid fractures: a case study. J Plast Reconstr Aesthet Surg 2009;62:e565-9.

5. Chen CT, Chen YR. Traumatic superior orbital fissure syndrome: current management. Craniomaxillofac Trauma Reconstr 2010;3:916.

6. Lee YB, Kim C, Park DH. Superior orbital fissure syndrome after repair of blow out fracture. J Korean Soc Plast Reconstr Surg 2011;38: 879-82.

7. Kim YJ, Choi WK. Delayed superior orbital fissure syndrome after reconstruction of blowout fracture. J Craniofac Surg 2016;27:e8-10.

8. Burdan F, Umlawska W, Dworzanski W, Klepacz R, Szumilo J, Staroslawska E, et al. Anatomical variances and dimensions of the superior orbital fissure and foramen ovale in adults. Folia Morphol (Warsz) 2011;70:263-71.

9. Morard M, Tcherekayev V, de Tribolet N. The superior orbital fissure: a microanatomical study. Neurosurgery 1994;35:1087-93. 